06,11

\title{
Электрические взаимодействия в смесях сегнетоэлектрических порошков хлорида диизопропиламмония и титаната свинца
}

\author{
(C) С.В. Барышников ${ }^{1,2}$, А.Ю. Милинский ${ }^{1}$ \\ ${ }^{1}$ Благовещенский государственный педагогический университет, \\ Благовещенск, Россия \\ ${ }^{2}$ Амурский государственный университет, \\ Благовещенск, Россия \\ E-mail: svbar2003@list.ru, a.milinskiy@mail.ru
}

Поступила в Редакцию 9 февраля 2021 г.

В окончательной редакции 9 февраля 2021 г.

Принята к публикации 12 февраля 2021 г.

Представлены результаты исследований фазовых переходов в смесях порошков $\mathrm{C}_{6} \mathrm{H}_{16} \mathrm{NCl}$ (DIPAC) с $\mathrm{PbTiO}_{3}$ методом дифференциального термического анализа (ДТА). Измерения проводились в режиме нагрева и охлаждения в интервале от 300 до $440 \mathrm{~K}$. Обнаружено, что электрические взаимодействия между частицами сегнетоэлектриков приводят к появлению дополнительных фазовых переходов для DIPAC в смесях порошков.

Ключевые слова: сегнетоэлектрик, смесь порошков, хлорид диизопропиламмония, DIPAC, фазовые переходы.

DOI: $10.21883 /$ FTT.2021.06.50938.023

\section{1. Введение}

Согласно существующим представлениям причиной сегнетоэлектрического состояния в кристаллах является дальнодействующее диполь-дипольное взаимодействие, которое отвечает за разбиение кристаллов на домены и влияние дипольных примесей на свойства сегнетоэлектриков [1-3]. Изучение кооперативных явлений в неупорядоченных системах показало, что введение нецентральных примесей в сильно поляризуемые матрицы может приводить к появлению сегнетоэлектрической фазы [2,3].

В отличие от сегнетоэлектрических кристаллов и их твердых растворов в композитах дипольные частицы расположены на значительных расстояниях. Однако, установлено, что и для таких систем возможно взаимное влияние компонентов на свойства друг друга. Для сегнетоэлектрических композитов $\left(\mathrm{KNO}_{3}\right)_{1-x} /\left(\mathrm{BaTiO}_{3}\right)_{x}$ и $\left(\mathrm{KNO}_{3}\right)_{1-x} /\left(\mathrm{KNbO}_{3}\right)_{x}$ наблюдается расширение температурной области существования сегнетоэлектрической фазы нитрата калия [4,5]. Для композитов $\left(\mathrm{NaNO}_{2}\right)_{1-x} /\left(\mathrm{BaTiO}_{3}\right)_{x}$ эффекты взаимодействия приводят к увеличению температурного диапазона существования несогласованной фазы нитрита натрия [6]. В [7] было обнаружено, что эффекты взаимодействия в смесях порошков DIPAB $\left(\mathrm{C}_{6} \mathrm{H}_{16} \mathrm{NBr}\right)$ и $\mathrm{PbTiO}_{3}$ приводят к появлению дополнительных фазовых переходов для DIPAB.

Целью настоящей работы являлось изучение взаимодействия частиц сегнетоэлектриков в смесях порошков DIPAC и $\mathrm{PbTiO}_{3}$. Исследование фазовых переходов в смесях проводилось методом дифференциального термического анализа (ДТА).

\section{2. Образцы иметодика эксперимента}

При комнатной температуре хлорид диизопропиламмония $\left(\mathrm{C}_{6} \mathrm{H}_{16} \mathrm{NCl}\right)$ кристаллизуется с образованием моноклинной кристаллической решетки с хиральной пространственной группой $P 2_{1}$. Его сегнетоэлектрическая активность наблюдается ниже $T_{c} \approx 440 \mathrm{~K}$. Спонтанная поляризация $P_{s}$ составляет $\approx 8.9 \mu \mathrm{C} / \mathrm{cm}^{2}$, коэрцитивное поле $E_{c} \approx 9.0 \mathrm{kV} \cdot \mathrm{cm}^{-1}$. Выше $T_{c} \mathrm{C}_{6} \mathrm{H}_{16} \mathrm{NCl}$ имеет кристаллическую структуру с пространственной группой $2 / m[8]$.

В нашей работе хлорид диизопропиламмония был получен реакцией диизопропиламина с 30\%-ным водным раствором $\mathrm{HCl}$ с последующей перекристаллизацией из метилового спирта. Согласно рентгеновскому спектру, полученный хлорид диизопропиламмония при комнатной температуре находился в полярной фазе $P 2_{1}$, что соответствует карточке ICDD 00-009-0589. Наблюдаемый фазовый переход является переходом первого рода и имеет $T_{c} \approx 439 \mathrm{~K}$ при нагреве и $\approx 435 \mathrm{~K}$ при охлаждении.

Титанат свинца является классическим сегнетоэлектриком с температурой Кюри $T_{c} \approx 763 \mathrm{~K}$. При комнатной температуре $\mathrm{PbTiO}_{3}$ находится в тетрагональной сегнетоэлектрической фазе с $P_{\mathrm{s}} \sim 60 \mu \mathrm{C} / \mathrm{cm}^{2}$ [9]. Для получения образцов использовались порошки DIPAC и $\mathrm{PbTiO}_{3}$ с частицами размером $\sim 15-20 \mu \mathrm{m}$ и $\sim 1 \mu \mathrm{m}$ соответственно. Порошки DIPAC и $\mathrm{PbTiO}_{3}$ смешивались в соответствующих пропорциях $(5,10,20,30$ и 40 vol.\% $\left.\mathrm{PbTiO}_{3}\right)$ и тщательно перемешивались.

Для снятия ДТА сигнала полученных смесей порошков использовался синхронный термоанализатор Linseis STA PT 600. Исследования проводились в режиме 

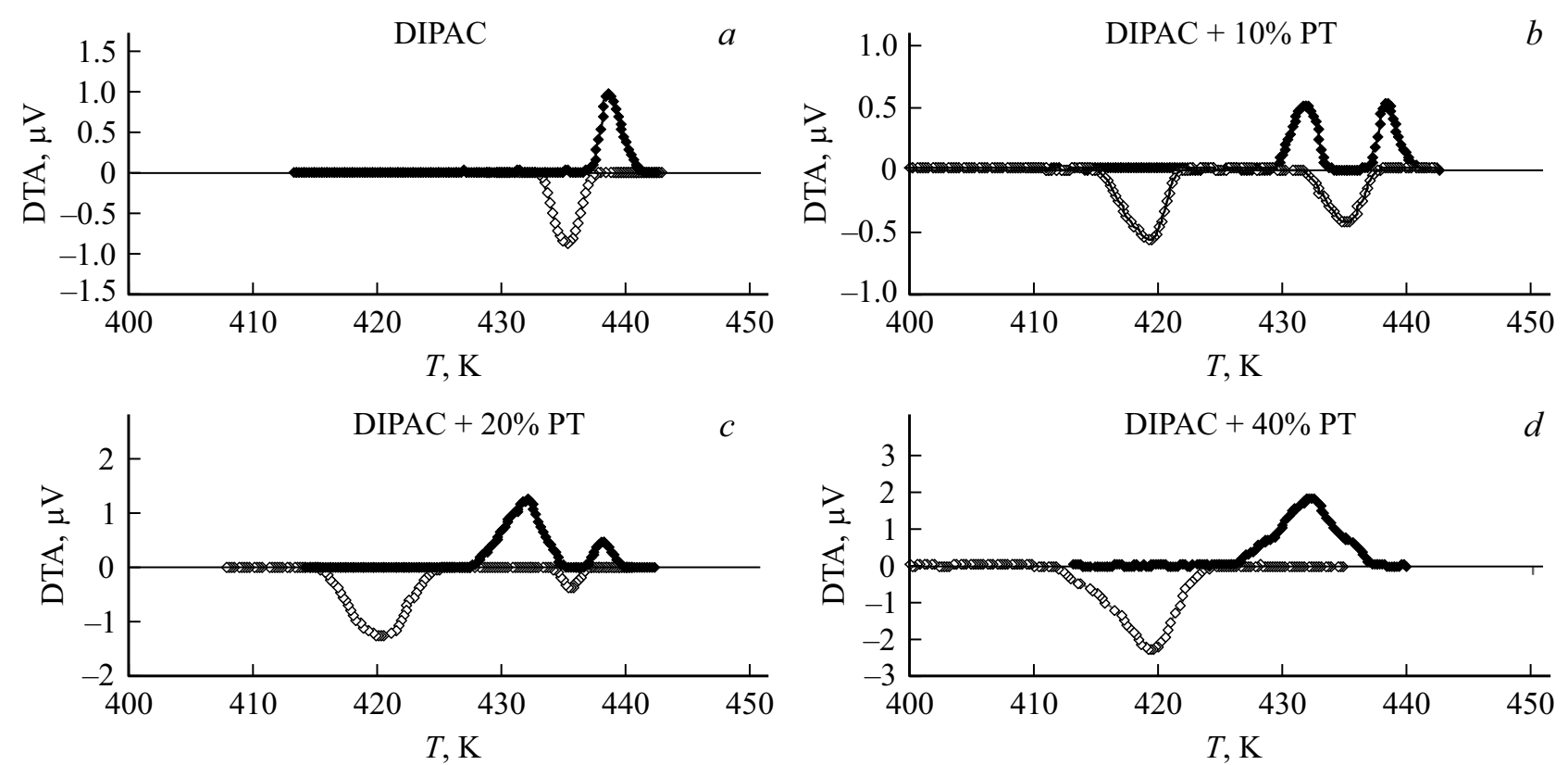

Рис. 1. ДТА - сигнал для смеси порошков DIPAC и $\mathrm{PbTiO}_{3}$ при $x=0(a), x=0.10(b), x=0.20(c)$ и $x=0.40(d)$. Положительный сигнал - нагрев, отрицательный - охлаждение.

нагрев-охлаждение со скоростью $2 \mathrm{~K} / \mathrm{min}$ в интервале температур от 300 до $440 \mathrm{~K}$.

\section{3. Экспериментальные результаты и обсуждение}

На рис. 1 показаны температурные зависимости сигнала ДТА для смеси порошков DIPAC и $\mathrm{PbTiO}_{3}$. Для $x$ от 0 до $\sim 0.05$ наблюдается один структурный переход $T_{c 1} \approx 439 \mathrm{~K}$ в режиме нагрева и $T_{c 2} \approx 435 \mathrm{~K}$ в режиме охлаждения. Температурный гистерезис обусловлен переходом первого рода. Увеличение содержания титаната свинца до $x \approx 0.1$ приводит к появлению дополнительных, более широких аномалий на кривых ДТА при $T_{c 3} \approx 432 \mathrm{~K}$ в режиме нагрева и при $T_{c 4} \approx 419 \mathrm{~K}$ в режиме охлаждения. Четыре аномалии на кривых ДТА четко наблюдаются до $x \approx 0.35$ и при содержании титаната свинца $x>0.4$ ДТА сигнал имеет аномалии только при температурах $T_{c 3}$ и $T_{c 4}$. На рис. 2 показана зависимость температур фазовых переходов DIPAC от доли $\mathrm{PbTiO}_{3}$ в смеси.

$\mathrm{C}$ ростом содержания $\mathrm{PbTiO}_{3}$ площадь под кривыми ДТА (соответствующая энтальпии перехода $[10]$ ) при температурах $T_{c 1}, T_{c 2}$ уменьшаются, а при $T_{c 3}$ и $T_{c 4}$ увеличиваются (рис. 3 ). Из результатов эксперимента следует, что появление фазовых переходов при $T_{c 3}$ и $T_{c 4}$ в DIPAC зависит от концентрации частиц $\mathrm{PbTiO}_{3}$ в смеси.

Для понимания полученных результатов рассмотрим массив малых частиц, находящихся на некотором расстоянии друг от друга. Для описания фазового перехода в отдельной частице обычно используется подход, ос-

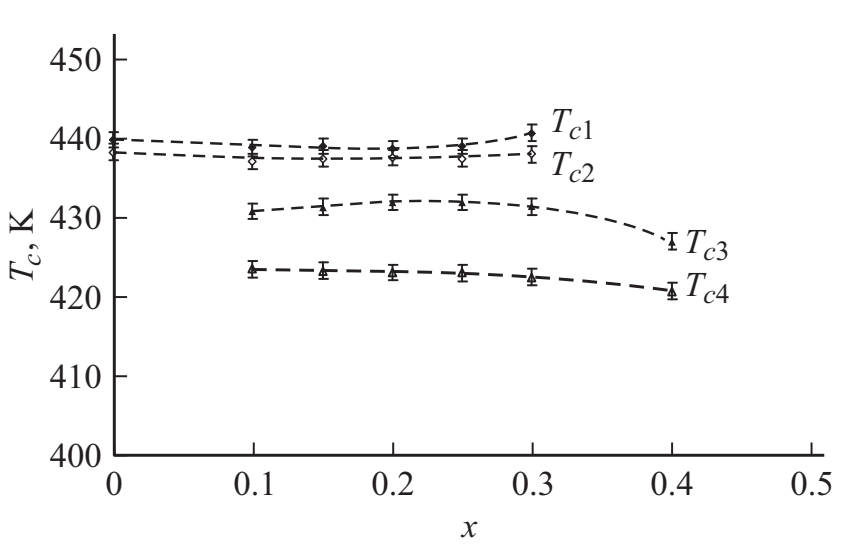

Рис. 2. Температуры фазовых переходов для смеси порошков DIPAC и $\mathrm{PbTiO}_{3}$ в зависимости от концентрации титаната свинца $x$, определенные по данным ДТА.

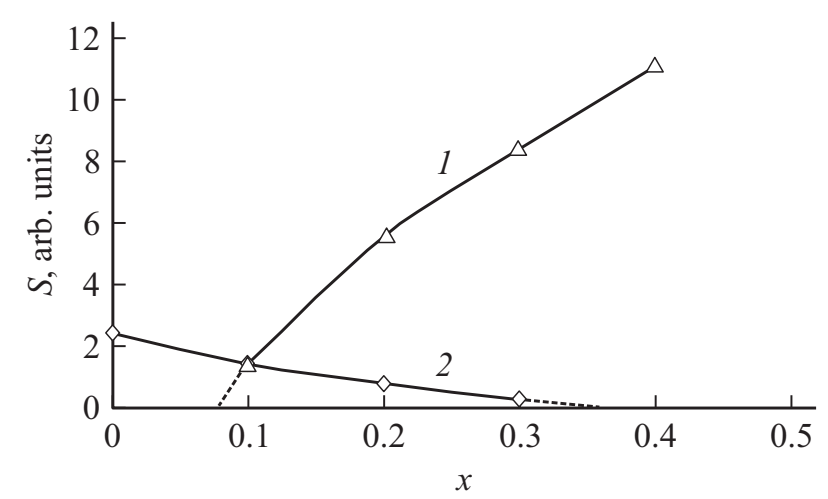

Рис. 3. Зависимость средней площади под кривыми ДТА при фазовых переходах с температурами $T_{c 1}, T_{c 2}$ (кривая 2) и температурами $T_{c 3}$ и $T_{c 4}$ (кривая 1 ). 
нованный на разложении Ландау [11], где поляризация играет роль параметра порядка. Таким образом, свободную энергию смеси сегнетоэлектрических частиц можно записать в виде:

$$
\begin{aligned}
& F=\sum_{i} \int_{V_{i}}\left(F_{0 i}+\frac{1}{2} \alpha_{i} P_{i}^{2}+\frac{1}{4} \beta_{i} P_{i}^{4}+\ldots\right) d \nu_{i} \\
& +\sum_{j} \int_{V_{j}}\left(F_{0 j}+\frac{1}{2} \alpha_{j} P_{j}^{2}+\frac{1}{4} \beta_{j} P_{j}^{4}+\ldots\right) d v_{j}+\sum_{i j} \Delta F_{i j},
\end{aligned}
$$

где $F_{0 i}$ и $F_{0 j}-$ вклады в свободную энергию, не зависящие от поляризации для первого и второго сегнетоэлектрика соответственно; $\alpha_{i}, \alpha_{j}, \beta_{i}, \beta_{j}$ - коэффициенты разложения Ландау; $P_{i}$ и $P_{j}-$ поляризация для частиц DIPAC и $\mathrm{PbTiO}_{3}$ соответственно; $\Delta F_{i j}$ - энергия взаимодействия между частицами, которая в случае порошков может иметь только электрическую природу. Если оценить напряженность электрического поля, создаваемого монодоменной частицей на ее поверхности по направлению поляризации, то получим $\sim 7 \cdot 10^{8} \mathrm{~V} / \mathrm{cm}$ для титаната свинца и $\sim 2.5 \cdot 10^{8} \mathrm{~V} / \mathrm{cm}$ для DIPAC. Доменная структура малых частиц $\mathrm{PbTiO}_{3}$ исследовалась в [12-13], где было показано, что с уменьшением размера частиц размер доменов уменьшается и при размере 30-20 nm происходит монодоменизация. Микронные частицы $\mathrm{PbTiO}_{3}$ не являются монодоменными, однако, обладают некоторым нескомпенсированным дипольным моментом, что следует из слипания частиц и перераспределения их при фазовом переходе. Данных по исследованию доменной структуры малых частиц DIPAC в публикациях пока нет.

Энергия электрического взаимодействия между дипольными частицами будет складываться из энергий Кеезома (энергия взаимодействия частиц с готовыми дипольными моментами) и Дебая (энергия взаимодействия дипольной и недипольной частиц, обусловленная наведенной поляризацией). При среднем размере частицы DIPAC $15-20 \mu \mathrm{m}$ и $\mathrm{PbTiO}_{3} 1 \mu \mathrm{m}$ одна сферическая частица DIPAC теоретически может контактировать приблизительно с 1000 сферическими частицами $\mathrm{PbTiO}_{3}$. На рис. 4 показана зависимость числа частиц $\mathrm{PbTiO}_{3}$, приходящихся на одну частицу DIPAC в смеси порошков в зависимости от $x$. На вставке рис. 4 приведены фрагменты электронных фотографий для $x=0.05$ и $x=0.3$, где наблюдается прилипание частиц $\mathrm{PbTiO}_{3}$ к частицам DIPAC.

Напряженность электрического поля на поверхности поляризованной частицы можно записать в виде [14]:

$$
E_{i}=\frac{P_{i} \cos \vartheta_{i}}{\varepsilon_{0}},
$$

где $P_{i}$ - некоторое усредненное значение поляризации, $\vartheta_{i}$ - угол между направлением поляризации и нормалью к поверхности. Задача о нахождении энергии электрического взаимодействия между сегнетоэлектрически-

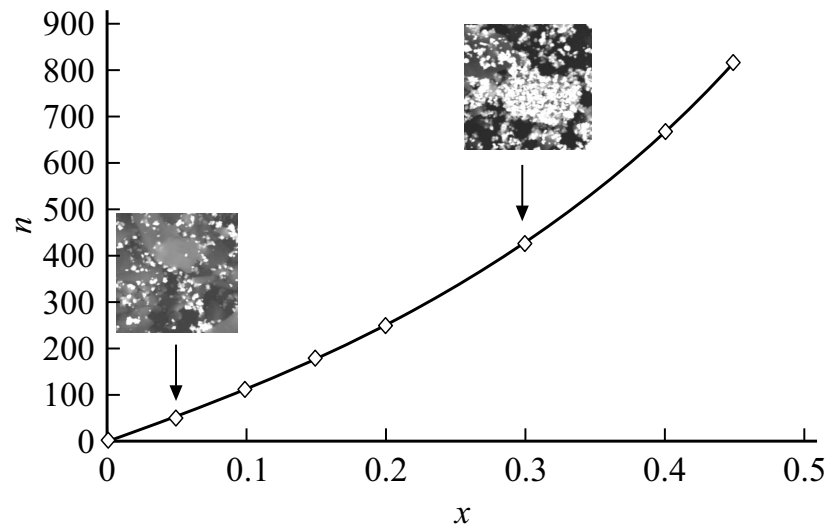

Рис. 4. Число частиц $\mathrm{PbTiO}_{3}$, приходящихся на одну частицу DIPAC в зависимости от $x$. На вставках показаны фрагменты электронных фотографий смеси порошков для $x=0.05$ и $x=0.3$.

ми частицами является задачей многих тел и не имеет точного решения. Однако, в общем виде изменение энергии одной частицы DIPAC в результате взаимодействия c $n$ ближайшими частицами $\mathrm{PbTiO}_{3}$ можно записать в виде:

$$
\begin{aligned}
\Delta F_{i}=\sum_{j=1}^{n} k_{i j} V_{j} \frac{P_{i} P_{j}}{\varepsilon_{0}} & +\sum_{j=1}^{n} k_{i j} V_{j} \frac{P_{i}^{2}}{\varepsilon_{0}^{2} \varepsilon_{j}} \\
& +\sum_{j=1}^{n} k_{i j} V_{j} \frac{P_{j}^{2}}{\varepsilon_{0}^{2} \varepsilon_{i}}+\ldots,
\end{aligned}
$$

где первое слагаемое - энергия Кеезома, описывающая взаимодействия частицы DIPAC с $n$ полярными частицами $\mathrm{PbTiO}_{3}$. Второе слагаемое - энергия Дебая, учитывающая взаимодействия частицы DIPAC с наведенными дипольными моментами в частицах $\mathrm{PbTiO}_{3}$. Третье слагаемое учитывает взаимодействие полярных частиц $\mathrm{PbTiO}_{3}$ с наведенным дипольным моментом в DIPAC. $k_{i j}$ - размерные коэффициенты, определяемые геометрией частиц и направлением поляризации, $V_{j}-$ объем $j$-той частицы $\mathrm{PbTiO}_{3}, V_{i}$ - объем частиц DIPAC, $\varepsilon_{i}$ и $\varepsilon_{j}$ - диэлектрические проницаемости частиц DIPAC и $\mathrm{PbTiO}_{3}$, соответственно.

Температуры фазовых переходов в частицах DIPAC будут определяться из условия минимума свободной энергии частиц с учетом взаимодействия:

$$
\begin{aligned}
\frac{d F_{i}}{d P_{i}} & =\frac{d}{d P_{i}}\left[\int_{V_{i}}\left(\frac{1}{2} \alpha_{i} P_{i}^{2}+\frac{1}{4} \beta_{i} P_{i}^{4}+\ldots\right) d \nu_{i}+\sum_{j} \Delta F_{i j}\right] \\
& =0
\end{aligned}
$$

Из соотношений (3) и (4) следует, что температуры фазовых переходов системы частиц в смеси по сравнению с температурой фазового перехода в системе частиц одного вида будут зависеть от концентрации и размеров 
частиц, величины их спонтанных поляризаций, степени поляризации частиц внедрения и их диэлектрической проницаемости.

Если проанализировать график зависимости температур фазовых переходов от объемного содержания титаната свинца (рис. 2), можно заключить, что в смеси присутствуют два состояния частиц DIPAC: первые не имеющие в ближайшем окружении поляризованных частиц $\mathrm{PbTiO}_{3}$ и их фазовые переходы $\left(T_{c 1}\right.$ и $\left.T_{c 2}\right)$ соответствуют фазовым переходам изолированных частиц DIPAC; вторые - соседствующие с дипольными частицами $\mathrm{PbTiO}_{3}$ и их температуры фазовых переходов $\left(T_{c 3}\right.$ и $\left.T_{c 4}\right)$ будут определяться с учетом энергии взаимодействия. Это следует из того, что площади под кривыми ДТА при $T_{c 3}$ и $T_{c 4}$ увеличиваются с ростом содержания частиц второго сорта, и, например, для $\mathrm{PbTiO}_{3}$ при $x \approx 0.4$ частиц первого сорта практически не остается.

\section{4. Заключение}

Как показали проведенные исследования, увеличение концентрации $\mathrm{PbTiO}_{3}$ в смеси порошков приводит к возникновению дополнительных фазовых переходов DIPAC при нагреве и охлаждении. Удельная теплота новых фазовых переходов растет с увеличением $x$. Появление дополнительных фазовых переходов можно объяснить электрическими взаимодействиями между частицами смеси.

\section{Финансирование работы}

Работа выполнена при поддержке Российского фонда фундаментальных исследований, грант № 19-29-03004.

\section{Конфликт интересов}

Авторы заявляют об отсутствии конфликта интересов

\section{Список литературы}

[1] V.A. Isupov. Phys. Solid State 45, 1107 (2003).

[2] B.E. Vugmeister, M.D. Glinchuk. Sov. Phys. JETP. 52, 482 (1980).

[3] B.E. Vugmeister, M.D. Glinchuk. Sov. Phys. Usp. 28, 589 (1985).

[4] E.V. Stukova, S.V. Baryshnikov. Inorg. Mater.: Appl. Res. 2, 434 (2011).

[5] E.V. Stukova, S.V. Baryshnikov. Perspekt. Mater. 13, 801 (2011).

[6] S.V. Baryshnikov, E.V. Stukova, E.Yu. Koroleva. Composites B 66, 190 (2014).

[7] С.В. Барышников, А.Ю.. Милинский. ФТТ 62, 1855 (2020).

[8] D.-W. Fu, W. Zhang, H.-L. Cai, J.-G. Ge, Y. Zhang, R.G. Xiong. Adv. Mater. 23, 5658 (2011).

[9] М. Лайнс, А. Гласс. Сегнетоэлектрики и родственные им материалы. / Пер. с англ. под ред. В.В. Леманова, Г.А. Смоленского. Мир, М. (1981). 736 с
[10] У. Уэндландт. Термические методы анализа. Мир, М. (1978). $527 \mathrm{c}$.

[11] V.L. Ginzburg. Phys. Usp. 44, 1037 (2001).

[12] W.L. Zhong, Y.X. Wang, C.L. Wang, B. Jiang, L.A. Bursill. Ferroelectrics 252, 11 (2001).

[13] B. Jiang, L.A. Bursill. Phys. Rev. B 60, 9978 (1999).

[14] Л.Д. Ландау, Е.М. Лифшиц. Электродинамика сплошных сред. Наука, М. (1982). 621 с.

Редактор Д.В. Жуманов 